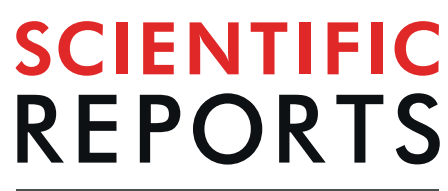

natureresearch

Check for updates

\title{
Rosmarinic Acid Exhibits Anticancer Effects via MARK4 Inhibition
}

\author{
Saleha Anwar ${ }^{1,7}$, Anas Shamsi ${ }^{1,7}$, Mohd Shahbaaz ${ }^{2,3}$, Aarfa Queen ${ }^{1,4}$, Parvez Khan ${ }^{1}{ }^{1}$, \\ Gulam Mustafa Hasan ${ }^{5}$, Asimul Islam ${ }^{1}{ }^{1}$, Mohamed F. Alajmi ${ }^{6}$, Afzal Hussain ${ }^{6}$, Faizan Ahmad ${ }^{1}$ \\ \& Md. Imtaiyaz Hassan $\mathbb{1}^{1 凶}$
}

Microtubule affinity regulating kinase (MARK4) is a potential drug target for different types of cancer as it controls the early step of cell division. In this study, we have screened a series of natural compounds and finally identified rosmarinic acid (RA) as a potential inhibitor of MARK4. Molecular docking and $500 \mathrm{~ns}$ all-atom simulation studies suggested that RA binds to the active site pocket of MARK4, forming enough number of non-covalent interactions with critical residues and MARK4-RA complex is stable throughout the simulation trajectory. RA shows an excellent binding affinity to the MARK4 with a binding constant $(K)$ of $10^{7} \mathrm{M}^{-1}$. Furthermore, RA significantly inhibits MARK4 activity $\left(\mathrm{IC}_{50}=6.204 \mu \mathrm{M}\right)$. The evaluation of enthalpy change $(\Delta H)$ and entropy change $(\Delta S)$ suggested that the MARK4-RA complex formation is driven by hydrogen bonding and thus complexation process is seemingly specific. The consequence of MARK4 inhibition by RA was further evaluated by cell-based tau-phosphorylation studies, which suggested that RA inhibited the phosphorylation of tau. The treatment of cancer cells with RA significantly controls cell growth and subsequently induces apoptosis. Our study provides a rationale for the therapeutic evaluation of RA and RA-based inhibitors in MARK4 associated cancers and other diseases.

Protein kinases are key regulators of signaling pathways and their abnormal expression is directly associated with cancer, neurodegenerative and other metabolic diseases ${ }^{1}$. Because of their important role in cancer, diabetes, and other neurodegenerative disorders, kinases are generally targeted for the development of new therapeutic molecules ${ }^{2,3}$. MARK4 is a member of Ser/Thr kinase, implicated as a drug target for cancer ${ }^{4}$, neurodegenerative disorders $^{5}$, diabetes ${ }^{6}$, obesity and other diseases ${ }^{7}$. Initially, the MARK kinases were recognized by their potential role to phosphorylate a serine motif in the microtubule-binding domain of tau; this phosphorylation plays a critical role in microtubule dynamics ${ }^{8}$.

The importance of MARK4 is described in recent reports which highlighted its role in different diseases viz. over-expression of MARK4 is associated with obesity and diabetes, Alzheimer's diseases (AD) and metastatic breast carcinomas ${ }^{9-11}$. The tissues associated with the highest expression of MARK4 are brain, kidney and testes $^{12,13}$. MARK4 expression is functionally associated with many signaling pathways such as NF- $\kappa B^{11}, m^{2} O^{14}$, $\mathrm{Wnt}^{10}$ and $\mathrm{Akt}^{14}$.

Overexpression of MARK4 is involved in cancer progression, metastasis, guiding neuronal migration, cell polarity, microtubule dynamics, apoptosis, cell cycle regulation, cell signaling and differentiation ${ }^{4,15,16}$. MARK4 plays an important role in breast cancer proliferation and migration through hippo signaling ${ }^{4}$. In addition, MARK4 regulates miR- 515-5p, that found to be involved in breast cancer cell proliferation and migration ${ }^{17}$. MARK4 is implicated in the regulation of dynamic biological functions like glucose and energy homeostasis ${ }^{18}$. It was found that MARK4 induces adipogenesis in adipocytes thereby stimulating apoptosis by the JNK1 pathway, showing the involvement of MARK4 with this important pathway ${ }^{19}$. All these studies indicate a vital role of MARK4 in the occurrence of diseases and thus targeted for the development of therapeutic molecules to address cancer $^{20,21}$ as well as neurodegenerative diseases ${ }^{12,22}$.

${ }^{1}$ Centre for Interdisciplinary Research in Basic Sciences, Jamia Millia Islamia, Jamia Nagar, New Delhi, 110025, India. ${ }^{2}$ South African Medical Research Council Bioinformatics Unit, South African National Bioinformatics Institute, University of the Western Cape, Private Bag X17, Bellville, Cape Town, 7535, South Africa. ${ }^{3}$ Laboratory of Computational Modeling of Drugs, South Ural State University, 76 Lenin Prospekt, Chelyabinsk, 454080, Russia. ${ }^{4}$ Department of Chemistry, Jamia Millia Islamia, Jamia Nagar, New Delhi, 110025, India. ${ }^{5}$ Department of Biochemistry, College of Medicine, Prince Sattam Bin Abdulaziz University, P.O. Box 173, Al-Kharj, 11942, Kingdom of Saudi Arabia. ${ }^{6}$ Department of Pharmacognosy, College of Pharmacy, King Saud University, Riyadh, Saudi Arabia. ${ }^{7}$ These authors contributed equally: Saleha Anwar and Anas Shamsi. ${ }^{\circledR e}$-mail: mihassan@jmi.ac.in 
Many natural compounds and their derivatives are being investigated for their curative properties for the development of new drugs ${ }^{16,23-26}$. In search of new drugs, natural compounds are explored due to their enormous structural and chemical diversity coupled with their clinical potential ${ }^{27,28}$. Many natural compounds possess anticancer or antioxidant properties and are being employed for drug discovery ${ }^{29-32}$. Rosmarinic acid (RA) is a naturally occurring phenolic compound, an ester of caffeic acid and 3,4-dihydroxy phenyl lactic acid, generally found in the plants of Lamiaceae (the mint) family. RA has been well studied for different biological activities including antioxidant ${ }^{33}$, anti-inflammator $y^{34}$, antiallergic ${ }^{35}$, anticancer ${ }^{36}$, antimicrobial ${ }^{37}$ and neuroprotective ${ }^{38}$ activities. RA is known to suppress various cancer types by interfering with the signaling pathways involved in the up-regulation of metastasis like ERK, a major factor in MAP kinases cascade is targeted by RA ${ }^{39,40}$. Phosphorylated ERK leads to an increase in COX-2 activity which has a major role in colorectal cancer ${ }^{39,41,42}$. Figure S1C shows the structure of RA.

Our study, for the first time, reports the binding mechanism and inhibitory efficacy of RA to the MARK4. Initially, molecular docking studies were employed to evaluate the binding affinity of RA with MARK4 followed by $500 \mathrm{~ns}$ MD simulation. In silico findings were validated by in vitro studies including cell-free and cell-based enzyme assay, suggesting the inhibition of MARK4 by RA. Further, fluorescence binding studies, isothermal titration calorimetry (ITC) and apoptosis studies suggested a high binding affinity of RA with MARK4 which subsequently induces apoptosis in MARK4 overexpressing cancer cells.

\section{Materials and Methods}

Chemicals and reagents. RA was purchased from Sigma-Aldrich Co. (St. Louis, MO, USA). The bacterial culture medium, Difco LB broth Miller (Luria-Bertani), was purchased from Becton, Dickinson and Company, Sparks, MD, USA. The Ni-NTA resin column and gel filtration column (Superdex-75) were obtained from GE Healthcare (GE Healthcare Life Sciences, Uppsala, Sweden. All other chemicals required for buffer preparation were obtained from Himedia (India). Double distilled and de-ionized water from a Milli-Q ${ }^{\circledR} U F-P l u s$ purification system was used for the preparation of all buffers. Dulbecco's modified eagle's media (DMEM), antibiotic antimycotic cocktail (penicillin, streptomycin, and amphotericin-B), fetal bovine serum (FBS), Phospho-Tau, MARK4 (MA5-27002) and actin monoclonal antibodies, MTT (3-[4,5-dimethylthiazol-2-yl]-2,5-diphenyltetrazolium bromide) and cell detachment enzyme (TrypLE express) were taken from Gibco-life technologies, Thermo Fisher Scientific (USA). Human adenocarcinoma alveolar basal epithelial cells (A549), breast cancer cells (MDA-MB-231), human neuroblastoma cells (SH-SY5Y) and human embryonic kidney cells (HEK293) were obtained from National Centre for Cell Sciences, Pune-411007, India.

Molecular docking analysis. DELL ${ }^{\circledR}$ Workstation with $4 \times 2.13 \mathrm{GHz}$ processor, $64 \mathrm{~GB}$ RAM and two TB hard disks running on Ubuntu 14.04.5 LTS operating system was retorted for molecular docking analysis. Bioinformatics tools AutoDock Vina ${ }^{43}$, Discovery studio ${ }^{44}$ and PyMOL ${ }^{45}$ were employed for docking and visualization purposes. Atomic coordinates of MARK4 crystal structure was taken from the Protein Data Bank (PDB ID: $5 E S 1)$ and refined further ${ }^{46,47}$. Docking was structurally blind for the compound where it was free to be in motion and search the binding site(s) of the protein. In total, nine docked conformations were obtained, out of which one having maximum binding affinity was selected.

MD simulations. MARK4 and MARK4-RA complex were subjected to MD simulation using GROMACS version 2018-2 ${ }^{48}$. Primarily, the GROMOS96 53a6 force-field ${ }^{49}$ was used for the generation of topologies of protein structure in the docking based generated complexes. The topologies of the studied ligand compound were generated using the PRODRG server ${ }^{50}$. Since PRODRG server does not contain the functionality of generating the partial charges of the RA; therefore, the DFT method implemented in GAUSSIAN which utilized the B3LYP 6-31 G (d,p) basis set and the CHELPG program ${ }^{51}$ was used for the charge correction. After successful topology generation of the docked complexes, they were solvated using the SPC/E water model ${ }^{52}$ and then neutralized by adding a suitable number of $\mathrm{Na}^{+}$and $\mathrm{Cl}^{-}$counter ions. Consequently, the system was subjected to energy minimization step using combined steepest descent as well as conjugate gradient algorithms, with a convergence criterion of $0.005 \mathrm{kcal} / \mathrm{mol}$. Before the equilibration step, the position restraints were applied to the structure of the ligand in the minimized system. The equilibration step was carried out into the combined stages of NVT (constant volume) and NPT (constant pressure) ensemble conditions, each at $100 \mathrm{ps}$ time scale. The temperature of $300 \mathrm{~K}$ was maintained for the system using the Berendsen weak coupling method, and the pressure of 1 bar was maintained utilizing Parrinello-Rahman barostat in the equilibration stage. The LINCS algorithm was used for the generation of the final conformational production stage for $500 \mathrm{~ns}$ timescale, and trajectories were generated, which were analyzed to understand the behavior of each complex in the explicit water environment. The changes in the Protein-ligand distance, H-bonds, RMSD, $R_{\mathrm{g}}$, RMSF, PCA and free energy landscapes of the complex system were analyzed. Furthermore, the Molecular mechanics Poisson-Boltzmann surface area (MM-PBSA) protocols implemented in g_mmpbsa package ${ }^{53}$ was used for the calculation of the free energy of binding protein and ligand molecules.

Expression and purification of MARK4. Human MARK4 was cloned, expressed, and purified as per our published protocol ${ }^{54,55}$. Further, the quality of isolated protein was investigated by kinase assay and purity was checked by SDS-PAGE and MARK4 protein was further confirmed with the help of Western blot using peptide-specific primary antibodies ${ }^{56}$.

Enzyme inhibition assay. To see the inhibitory effect of RA on MARK4, an ATPase assay was carried out in the presence of increasing concentrations of RA. Freshly prepared ATP $(200 \mu \mathrm{M})$ was added to MARK4 $(4 \mu \mathrm{M})$ and final reaction mixture of $100 \mu \mathrm{l}$ was incubated at $25^{\circ} \mathrm{C}$ for 1 hour. Malachite green $(200 \mu \mathrm{l})$ was further added to the reaction mixture to stop the reaction followed by incubation of samples at room temperature for 
20-25 minutes for the development of color. From the final reaction mixture, $100 \mu \mathrm{l}$ was transferred to a 96-well plate in triplicates to measure spectrophotometrically at $620 \mathrm{~nm}$.

Steady-state fluorescence studies. Fluorescence quenching experiments were carried out on Jasco FP-6200 spectrofluorometer attached with an external water bath which serves as a temperature controller. The experiment was carried out at three different temperatures in triplicates. The protein concentration was kept constant at $4 \mu \mathrm{M}$ while the ligand was titrated in the ratio of $1: 9$ at all three temperatures $(288 \mathrm{~K}, 298 \mathrm{~K}$, and $305 \mathrm{~K})$. The fluorescence intensities were corrected for the inner filter effect according to Chi \& Liu, $2011^{57}$ the following equation:

$$
F_{\text {corrected }}=F_{\text {observede }}\left(A_{e x}+A_{\text {obs }}\right) / 2
$$

$F_{\text {corrected }}$ and $F_{\text {observed }}$ are the corrected and observed fluorescence intensities respectively.

$A_{e x}$ and $A_{o b s}$ are the absorption of the system at excitation and emission wavelengths, respectively. The fluorescence intensities were corrected after taking filter effect into account and all the reported spectra are the subtracted spectra taking fluorescence of ligand alone. Fluorescence quenching data was further analyzed employing Stern-Volmer, modified Stern-Volmer and van't Hoff equation.

Isothermal titration calorimetry. ITC measurements were done at $25^{\circ} \mathrm{C}$ on a VP-ITC microcalorimeter from MicroCal, Inc (GE, MicroCal, USA). The first injection was a false one of $2 \mathrm{~s}$ with other injections set at $10 \mathrm{~s}$. The sample cell was filled with MARK4 with reference cell with a reference buffer and a syringe containing $500 \mu \mathrm{M}$ RA was titrated into MARK4. MicroCal Origin 8.0 was used to analyze the obtained data.

Cell culture and cytotoxicity studies. SH-SY5Y cells were grown in a 1:1 mixture of Eagle's minimum essential medium and F12 medium, A549 cells were maintained in F12K, HEK293 and MCF10A cells were grown in DMEM cell growth medium (having $10 \%$ heat-inactivated FBS and $1 \%$ antibiotic-antimycotic solution) in a humidified $\mathrm{CO}_{2}$ incubator $\left(5 \% \mathrm{CO}_{2}, 37^{\circ} \mathrm{C}\right)$. The cytotoxicity studies of selected synthesized compounds were accessed using standard MTT assay ${ }^{16,58,59}$. Briefly, 5000-6000 cells/well were plated in 96-well cell culture plate and next day cells were treated with selected compounds $(0-200 \mu \mathrm{M})$ for $72 \mathrm{~h}$. After $72 \mathrm{~h}$ incubation, the culture medium was removed and after washing with phosphate buffer saline ( $\mathrm{pH} 7.4)$ cells were incubated with MTT solution (a mixture of $100 \mu \mathrm{l}$ in complete medium and $25 \mu \mathrm{l}$ of MTT solution taken from $5 \mathrm{mg} / \mathrm{ml} \mathrm{stock}$ ), at $37^{\circ} \mathrm{C}$ in the $\mathrm{CO}_{2}$ incubator. Afterward, the resultant formazan crystals were dissolved in 150-200 $\mu$ l of DMSO and the absorbance of the reaction product was measured at $570 \mathrm{~nm}$ using a multiplate ELISA reader (BioRad). The percentage of cell viability was calculated and plotted as a function of RA concentration. To nullify the DMSO effect, respective DMSO treatment was performed and subtracted from corresponding RA treatment groups, whereas for anticancer studies paclitaxel has been taken as a positive control.

Tau-phosphorylation assay. To see the effect of RA treatment on the activity of MARK4, Tau-phosphorylation studies were performed as per the previously published protocol ${ }^{20}$. Briefly, SH-SY5Y cells were incubated with $\mathrm{IC}_{50}$ and $2 \times \mathrm{IC}_{50}$ doses of RA. Approximately, $10^{6}$ cells were collected after $48 \mathrm{~h}$ treatment, washed with PBS, fixed and incubated with anti-tau antibodies at $25^{\circ} \mathrm{C}$ for $2-4 \mathrm{hrs}$. Following the incubation, cells were washed and analyzed by flow cytometry.

Protein isolation and western blot. MDA-MB-231 vehicle control or RA treated cells were lysed in RIPA cell lysis buffer (Thermo Fisher Scientific, USA). Total protein was isolated and quantified by Bicinchoninic acid assay kit. Approximately 30-40 $\mu \mathrm{g}$ of whole-cell protein was diluted with 6 X Laemmli's buffer, boiled for 3-5 min and resolved using 12\% SDS-polyacrylamide electrophoresis under reducing conditions. The resolved polypeptides were transferred to polyvinylidene fluoride (PVDF) membrane using blotting and identified using protein-specific primary antibodies and horseradish peroxidase conjugated secondary antibodies. Luminol was used as a chemiluminescent substrate for $\mathrm{HRP}^{56}$.

Cell apoptosis assay. Apoptosis inducing properties of RA was studied using Annexin-V/PI staining as described previousl ${ }^{58,60}$. In brief, cells were treated with $\mathrm{IC}_{50}$ and $2 \times \mathrm{IC}_{50}$ doses of RA for $72 \mathrm{~h}$ at $37^{\circ} \mathrm{C}$, while control cells were treated with cell culture media only or vehicle control. After $72 \mathrm{~h}$ incubation, $\sim 1.0-1.5 \times 10^{6}$ cells were collected, washed three times with PBS and stained using FITC labeled Annexin-V and PI (BD-Biosciences, USA). Stained cells were analyzed using BD LSR II Flow Cytometry Analyzer and FlowJo.

Statistical analysis. All the experiments were performed in triplicates and the data obtained has been expressed in mean \pm standard error of the mean (SEM).

\section{Results and Discussion}

Screening of natural products. Polyphenols are known for their diverse activities including, anticancer, antidiabetic, and neurodegerative diseases. Recently, we have reported that many natural products efficiently bind to MARK4 and subsequently inhibit its activity ${ }^{16,20}$. We have estimated the binding affinity of different natural products using molecular docking analysis (Table 1). Based on binding energy, residues involved in binding and binding affinity, the best compounds were screened out and subjected to enzyme inhibition assay (Fig. SI.1B, Table 1). Based on molecular docking analysis, enzyme inhibition potential and binding affinity, RA was selected as the best inhibitor of MARK4.

Molecular docking studies. Molecular docking analysis revealed that RA occupies the active site of MARK4 with a significantly high binding affinity of $-8.1 \mathrm{kcal} / \mathrm{mol}$ and forming strong interactions with the 


\begin{tabular}{|c|c|c|c|c|}
\hline S. No. & Polyphenol & Structure & $\begin{array}{l}\Delta G(\mathrm{kcal} / \\
\mathrm{mol})\end{array}$ & $\begin{array}{l}\text { Binding constant } \\
(K) M^{-1}\end{array}$ \\
\hline 1. & Gallic Acid & & -5.4 & $0.7 \times 10^{1}$ \\
\hline 2. & Caffeic Acid & & -5.8 & $0.9 \times 10^{1}$ \\
\hline 3. & Ferulic Acid & & -5.9 & $0.5 \times 10^{2}$ \\
\hline 4. & Ellagic Acid & & -8.5 & $1.01 \times 10^{2}$ \\
\hline 5. & Quercetin & & -7.9 & $1.025 \times 10^{2}$ \\
\hline 6. & Vanillin & & -5.3 & $1.61 \times 10^{5}$ \\
\hline 7. & Rosmarinic acid & & -8.1 & $0.5 \times 10^{7}$ \\
\hline
\end{tabular}

Table 1. Binding parameters of polyphenols with MARK4 obtained from fluorescence and docking studies. ${ }^{*} \Delta G$ calculated from molecular docking. ${ }^{~} K$ calculated from fluorescence quenching studies performed and reported as per our previous studies.

functionally important residues, Glu133, Ala135, Ile62, Lys85 and Asp196 (Fig. 1A). This high binding affinity is attributed to the formation of a great number of hydrogen bonds to the catalytic domain of MARK4 (Fig. 1). In addition, several van der Waals interactions were offered by the Gly63, Phe67, Val70, Ala83, Val116, Met132, Tyr134, Glu139, Glu182, Leu185 and Ala195 of MARK4 (Fig. 1B). It was quite apparent from the reported crystal structure of MARK4 that its known inhibitor binds to the Asp $196^{47}$. Recent studies showed that many other natural compounds occupied the same cavity of MARK4 with a higher binding affinity ${ }^{16,20}$. We observed that the number of hydrogen bonds offered by MARK4 to RA is significantly very high and thus bearing excellent binding affinity.

MD simulation. To get insights into the interaction mechanism, we have performed an extensive MD simulation for both MARK4 and MARK4-RA complex for $500 \mathrm{~ns}$. RA assumed a close binding conformation with MARK4. RA was found in the reange of $0.15-0.25 \mathrm{~nm}$ and 6.78 average number of hydrogen bonds were present between the protein and RA during MD simulations (Fig. 2A,B). The stability of the system was further assessed using the calculated radius of gyration $\left(R_{\mathrm{g}}\right)$ and root mean square deviation (RMSD) values which showed that the system achieved the equilibrium conformation after $100 \mathrm{~ns}$ (Fig. 2C,D). There is a visible difference in the $R_{\mathrm{g}}$ and RMSD values of MARK4 and MARK4-RA complex indicating that the bound form shows an increased dynamic than the unbound form highlighting the perturbation effect of RA on the structure of MARK4.

The differences in the structrural conformation of MARK4 and MARK4-RA complex were further studied by analysing the free energy landscapes. A distinct difference in the free energies was observed between the two conformations (Fig. 3). In the case of MARK4, an energy favoured and relatively stable conformation was observed as compared to the MARK4-RA complex, indicating RA binding to MARK4 perturb its folding pattern and inhibit its function.

The active site of the MARK4 is having Val31, Ala44, Lys46, Glu94, Ala96, Gly99, Glu143, Asn144 and Asp157 residues. A significant difference in the conformation of these residues in MARK4 and MARK4-RA was observed (Fig. 4A). The active site residues form the corresponding $\beta 2, \beta 3, \beta 5$ and $\alpha 5$ of the secondary structure elements showed a relatively lower motion in the constituent residues in free MARK4, indicated the presence of lower relative energy as compared to the MARK4-RA complex. Furthermore, the flexibility of the conformational states of both MARK4-RA complex and only MARK4 were assessed using Principle Component Analysis (PCA). It is 
A

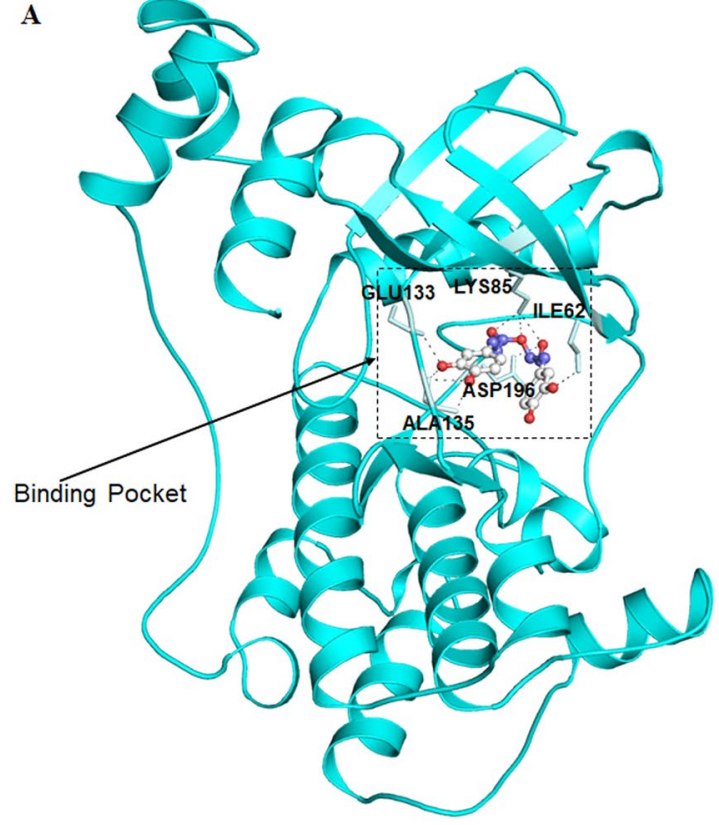

B

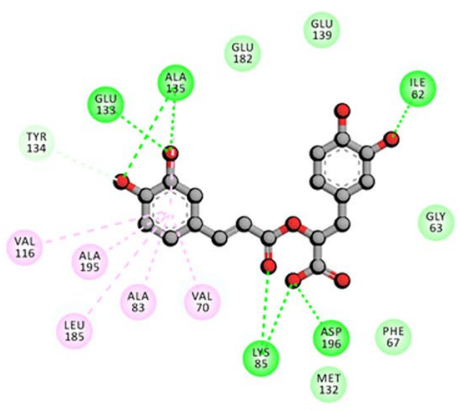

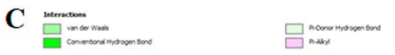

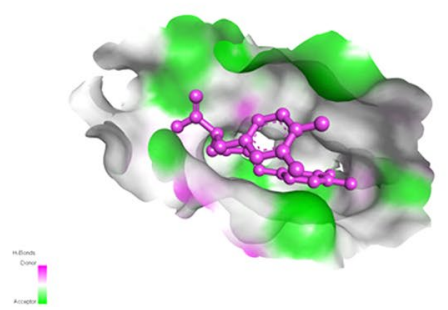

Figure 1. Molecular docking of RA with MARK4: (A) Cartoon representation of MARK4 with bound RA in the active site pocket with residues involved in polar interactions (stick model). (B) Two-dimensional representation of interactions involved between RA and MARK4. (C) RA molecule depicted by the ball and stick model in the binding pocket of MARK4.

A

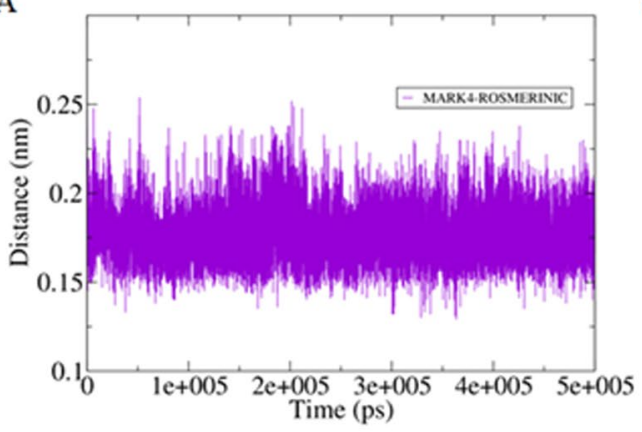

B

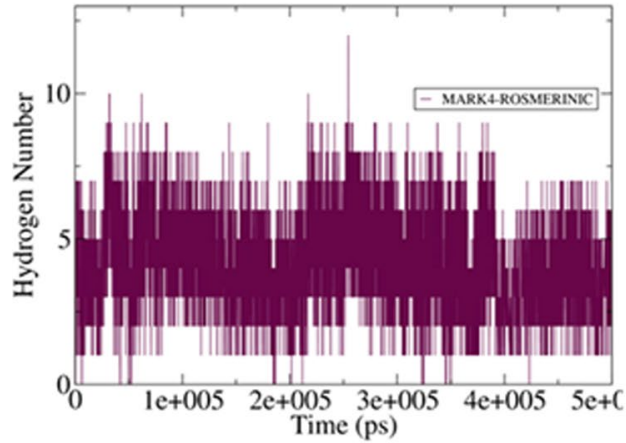

C

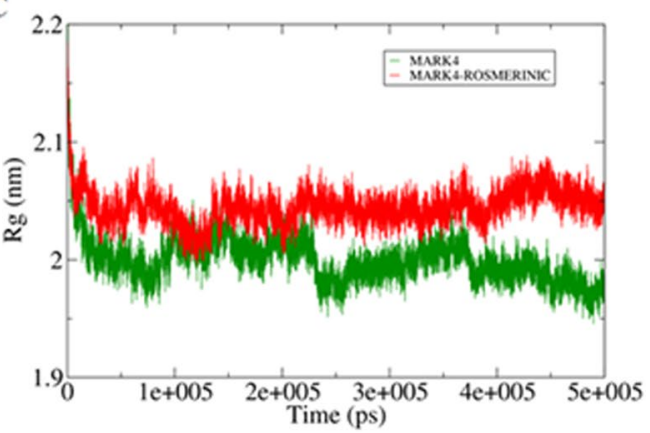

$\mathrm{D}$

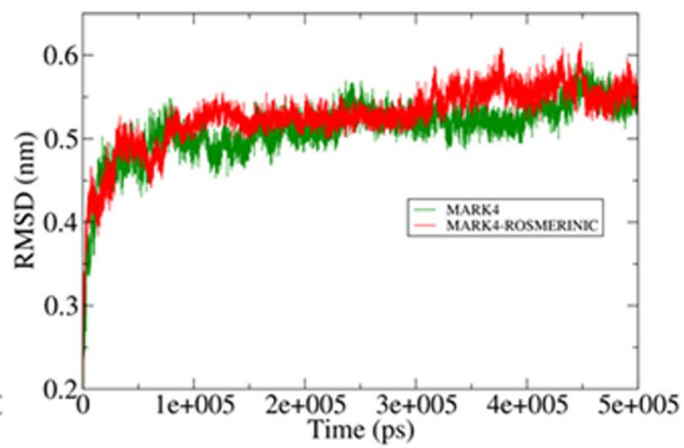

Figure 2. (A) Plot showing changes in the computed distance between the MARK4 and RA. (B) Hydrogen bond fluctuation curve highlighting the changes in the observed number. (C) The $R_{\mathrm{g}}$ curves showing the difference in the compactness between the RA bound and unbound MARK4. (D) The RMSD plot showing the changes between the stabilities in the observed systems. 
A

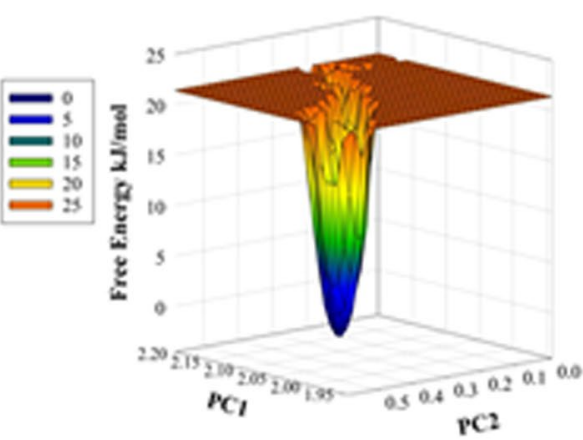

C
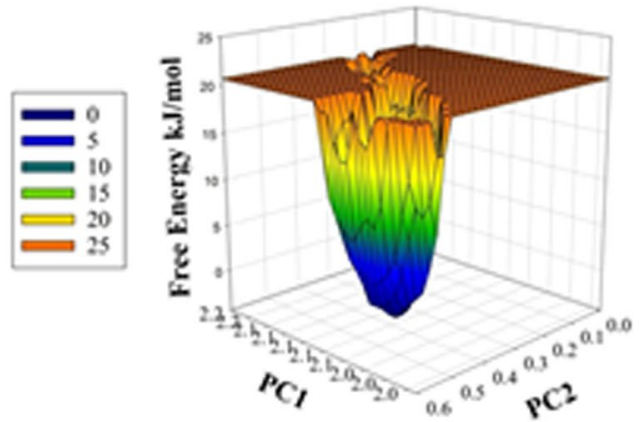

B

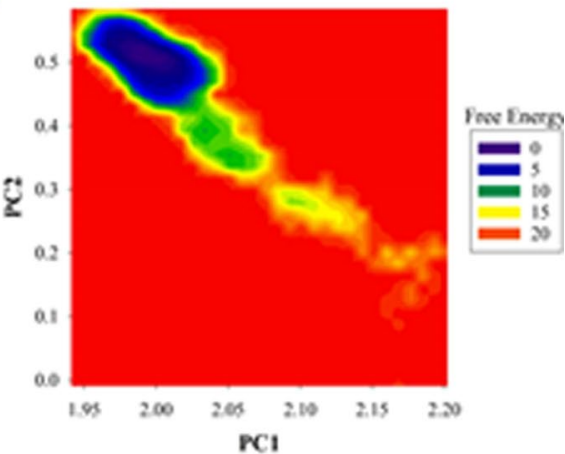

D

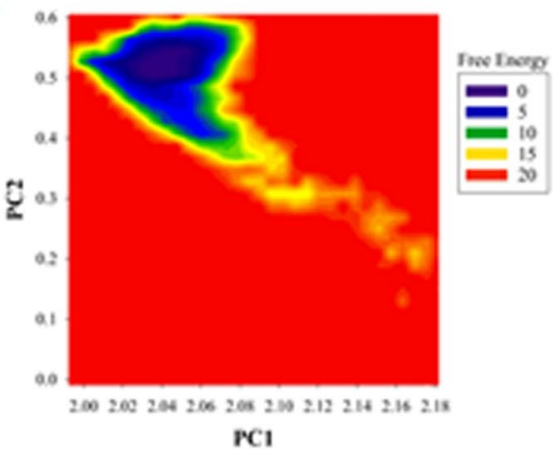

Figure 3. Plots of (A) free energy landscape and (B) Contour map for the of MARK4. (C and D) The graphical representation of the (C) free energy landscape and (D) Contour map for the of MARK4-RA.

a statistical technique used for the reduction of the data complexity and is significant in assessing the variation in the atomic motion in biomolecules in the course of MD simulations. A set of eigenvectors and eigenvalues were used to describe the motion of the protein atomic structure. The PCA is significant in the established correlation between the protein functionalities and conformation. RA-MARK4 complex occupies a larger conformational space as compared to the free MARK4, indicating higher structural stability in the unbound.

Further, MMPBSA based algorithm was used for the calculation of energies present between MARK4 and MARK4-RA docked complex (Fig. 4C). Total free energy of binding between the protein and the inhibitor was observed around $-600 \mathrm{~kJ} / \mathrm{mol}$ with electrostatic energy is the major contributor to the binding of the RA with MARK4. This observation validated the reliability of the RA binding to the MARK4.

Steady-state fluorescence binding studies. Intrinsic fluorescence is used to investigate the changes in the local microenvironment of aromatic amino acid residues and is an important tool to detect complex formation between protein and ligand ${ }^{61,62}$. To study the mechanism of quenching for MARK4-RA interaction, various parameters such as Stern-Volmer constant $\left(K_{\mathrm{sv}}\right)$, binding constant $(K)$ and the number of binding sites $(n)$ were estimated. The variation of binding parameters as a function of temperature may suggest the operative mode of interaction which can either be static or dynamic or a combination of both since the temperature dependence pattern of quenching parameters can differentiate between the dynamic and the static quenching. MARK4 was successfully cloned, expressedand purified. Figure S1A shows a single band in SDS-PAGE evident of purified MARK4. Steady-state fluorescence was carried out at three different temperatures, $288 \mathrm{~K}, 298 \mathrm{~K}$ and $305 \mathrm{~K}$. Figure $5(\mathrm{~A}-\mathrm{C})$ shows the fluorescence spectra of MARK4 before and after the addition of RA $(4-36 \mu \mathrm{M})$ at $288 \mathrm{~K}$, $298 \mathrm{~K}$ and $305 \mathrm{~K}$. MARK4 shows a peak of maxima around $344 \mathrm{~nm}$; RA was non-fluorescent under similar conditions (Curve not shown). We observed a gradual decrease of fluorescence emission in a dose-dependent manner, suggesting complex formation between MARK4 and RA.

When protein was excited at $295 \mathrm{~nm}$, only Trp fluorescence is considered whilst excitation at $280 \mathrm{~nm}$ considers tryptophan, tyrosine and phenylalanine. Both wavelengths were used in experiments to see if tryptophan is the key player or other fluorophores are also takes part in fluorescence. Figure 5D depicts that tryptophan is the key player involved as evident from fluorescence titration curves at $280 \mathrm{~nm}$ and $295 \mathrm{~nm}$ which nearly overlap each other. This overlapping suggests that tryptophan is actual fluorophore that plays major role.

To analyze the quenching data, Stern Volmer equation (Eq. 2) was deployed to find the Stern-Volmer constant:

$$
\frac{F_{0}}{F}=1+K_{s v}[C]
$$

where $F_{0} \& F$ are fluorescence intensity of MARK 4 in the absence and presence of RA, $[C]$ is the concentration of RA. $K_{\mathrm{sv}}$ refers to Stern-Volmer constant. 
A

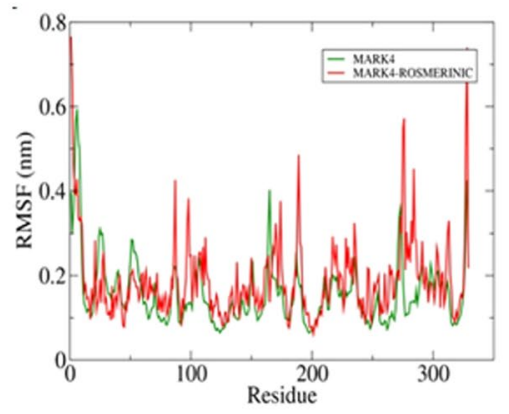

B

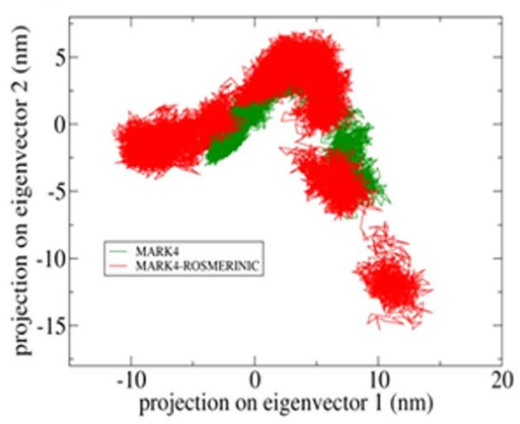

C

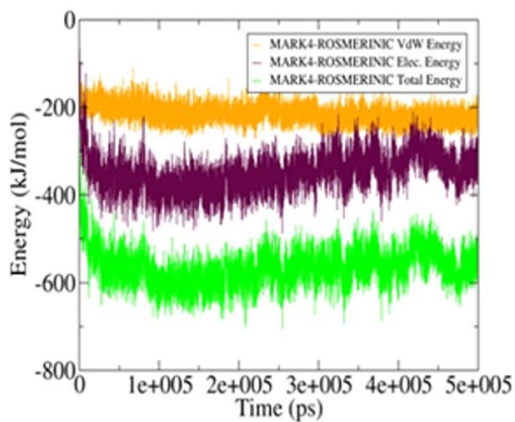

Figure 4. (A) The graphical representation of the changes observed in the fluctuation of the constituent residues between the RA bound and free MARK4 (B) 2-D eigenvector projection plot showing the differences between the flexibility of the two studied forms. (C) MMPBSA based generated curves highlighting the changes in the total, electrostatic and Van der Waals energies calculated between the MARK4 and RA.

A

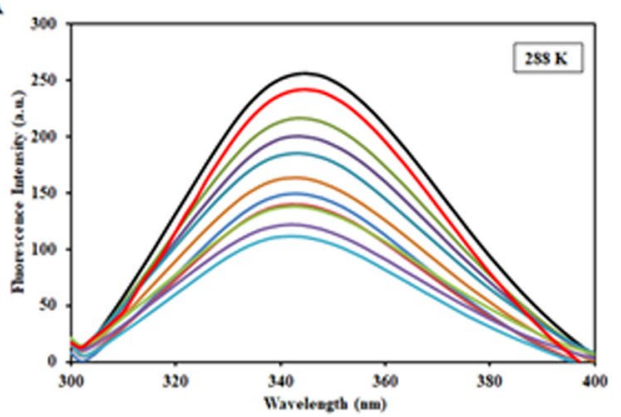

B

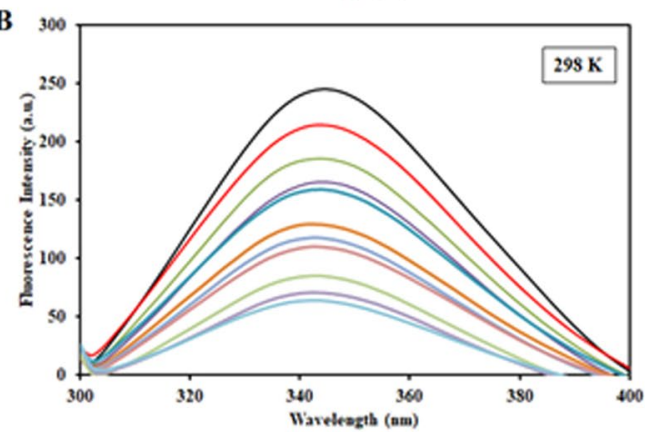

C

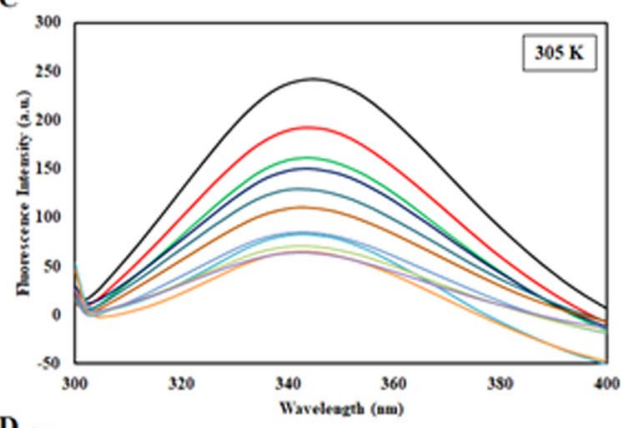

D

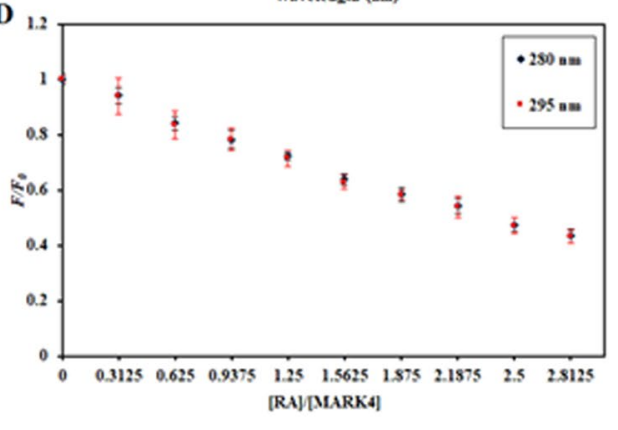

Figure 5. Steady-state fluorescence of MARK4 in the absence and presence of RA (4-36 $\mu \mathrm{M})$ at (A) $288 \mathrm{~K}$ and (B) $298 \mathrm{~K}$ and (C) $305 \mathrm{~K}$. (D) Fluorescence titration curves of the MARK4-RA system at an excitation

Figure 6A shows the Stern-Volmer plots of MARK4 quenching in the presence of RA. There was a positive deviation observed in the Stern-Volmer plot and hence, it can be assumed that both modes of quenching (static and dynamic) might be present. By fitting the fluorescence intensity ratio $F_{0} / F$ for different quencher concentration [C] (only linear points were considered) in Eq. 2, it gives us $K_{\mathrm{sv}} K_{\mathrm{sv}}$ was found at three different temperatures (Table 2).

The $K_{\mathrm{sv}}$ value decreases with increasing temperature for static quenching while the opposite effect is observed for dynamic quenching. The values of $K_{\mathrm{sv}}$ (Table 2) were found to increase with an increase in temperature. Thus, we can say that the MARK4-RA complex formation is driven by the dynamic mode of quenching. The mode of quenching was further confirmed from the value of bimolecular quenching rate constant, $K_{\mathrm{q}}$, which was calculated as per Eq. 3

$$
K_{q}=\frac{K_{s v}}{\tau_{0}}
$$

$\tau_{\mathrm{o}}$ refers to the average integral fluorescence lifetime of tryptophan and is reported to be $2.7 \times 10^{-9} \mathrm{~s}$. 


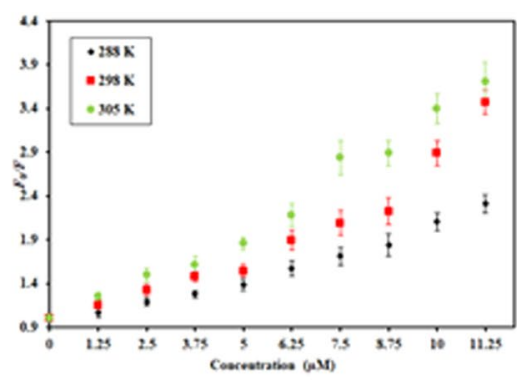

B

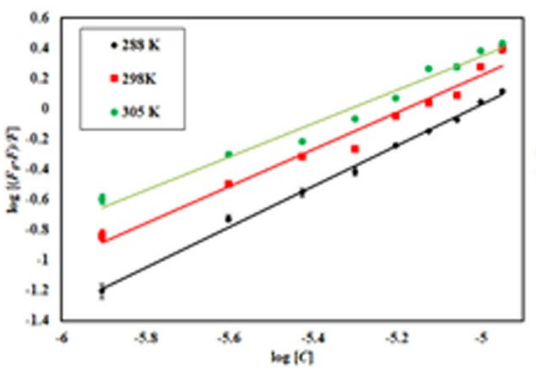

C

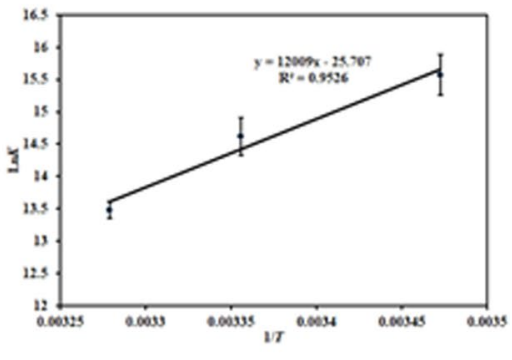

Figure 6. (A) Stern-Volmer plots of MARK4-RA system at three different temperatures $(288 \mathrm{~K}, 298 \mathrm{~K}$, and $305 \mathrm{~K})$. (B) Modified Stern-Volmer (double-log relation) plots of the MARK4-RA system at three different temperatures $((288 \mathrm{~K}, 298 \mathrm{~K}$, and $305 \mathrm{~K})$. (C) van't Hoff plot having a natural log of binding constants obtained at different temperatures on the $\mathrm{Y}$-axis against the inverse of the temperatures used for fluorescence quenching studies on the $\mathrm{X}$-axis.

\begin{tabular}{|l|l|l|l|}
\hline Temperature (K) & $\boldsymbol{K}_{s v}\left(\mathbf{1 0}^{\mathbf{5}} \mathbf{M}^{-\mathbf{1}}\right)$ & $\boldsymbol{K}_{\mathbf{q}}\left(\mathbf{1 0}^{\mathbf{1 4}} \mathbf{M}^{-\mathbf{1}} \mathbf{s}^{-\mathbf{1}}\right)$ & $\mathbf{R}^{\mathbf{2}}$ \\
\hline 288 & 11.5 & 4.25 & 0.97 \\
\hline 298 & 24.9 & 9.22 & 0.91 \\
\hline 305 & 29.4 & 10.88 & 0.98 \\
\hline
\end{tabular}

Table 2. Thermodynamic parameters of the MARK4-RA system as calculated from fluorescence quenching studies.

\begin{tabular}{|c|c|c|c|c|c|c|}
\hline Temperature (K) & $K\left(10^{7} M^{-1}\right)$ & $n$ & $\Delta G\left(\mathrm{kcal} \mathrm{mol}^{-1}\right)$ & $\Delta S\left(\mathrm{calmol}^{-1} \mathbf{K}^{-1}\right)$ & $\Delta H\left(\mathrm{kcal} \mathrm{mol}^{-1}\right)$ & $T \Delta S^{\circ}\left(\mathrm{kcal} \mathrm{mol}^{-1}\right)$ \\
\hline 288 & 0.5 & 1.34 & -8.95616 & \multirow{3}{*}{-41.82} & \multirow{3}{*}{-21.003} & -12.0468 \\
\hline 298 & 0.2 & 1.22 & -8.53787 & & & -12.4651 \\
\hline 305 & 0.07 & 1.10 & -8.24506 & & & -12.7579 \\
\hline
\end{tabular}

Table 3. Binding parameters and thermodynamic parameters for the MARK4-RA system.

The value of $\mathrm{Kq}$ for MARK4-RA interaction was substantially higher than the maximum dynamic quenching constant $\left(10^{10} \mathrm{M}^{-1} \mathrm{~s}^{-1}\right)$ suggesting a static mode of quenching to be operative for this interaction. Thus, MARK4-RA quenching is guided by a mixture of the static and dynamic modes of quenching.

Further, decrease in fluorescence in the presence of RA was analyzed by modified Stern-Volmer equation (Eq. 4$)^{63,64}$.

$$
\log \frac{F_{0}-F}{F}=\log K+n \log [C]
$$

Figure $6 \mathrm{~B}$ depicts the experimental data fitting as per the double log relation with the intercept of plot giving the binding constant. The value of binding constant $(K)$ (Table 2) was found to be $0.5 \times 10^{7} \mathrm{M}^{-1}, 0.2 \times 10^{7} \mathrm{M}^{-1}$, $0.07 \times 10^{7} \mathrm{M}^{-1}$ at 288,298 and $305 \mathrm{~K}$. These values suggested that RA has a very high binding affinity to MARK4. The value of $K$ was found to decrease with increasing temperature, (Table 3 ) suggesting that at a higher temperature, less stable complex was formed.

Thermodynamic analysis of MARK4-RA interaction. Using van't Hoff equation (Eq. 5$)^{65}$, thermodynamic parameters for ligand-protein interaction such as Gibbs free energy change $(\Delta G)$, enthalpy change $(\Delta H)$ and entropy change $(\Delta S)$ can be calculated.

$$
\Delta G=-R T \operatorname{Ln} K=\Delta H-T \Delta S
$$

$K$ is the binding constant, $\Delta H$ is enthalpy change, $\Delta G$ is Gibbs free energy change, $\Delta S$ is entropy change, and $R$ is the universal gas constant $\left(1.987 \mathrm{cal} \mathrm{mol}^{-1} \mathrm{~K}^{-1}\right)$. The experimental fitting of data as per Eq. 5 is shown in Fig. $6 \mathrm{C}$ ( $\ln K$ on $Y$-axis and $1 / T$ on $Y$-axis). The slope of this plot gives the value of $-\Delta H / R$, and the intercept giving the value of $\Delta S / R$."T".

These parameters can also tell about the various characteristics of the reaction such as the forces responsible for driving the reaction i.e. van der Waals, electrostatic, hydrophobic and hydrogen bonds. This reaction is spontaneous as suggested by the negative value of $\Delta G$ that was obtained employing Eq. $5^{66}$. Further, the negative values of $\Delta H$ and $\Delta S$ (Table 3) obtained from the slope and intercept of this plot suggested the reaction to be exothermic and driven by enthalpy and not entropy. These negative values of $\Delta H$ and $\Delta S$ further suggested that major 
A

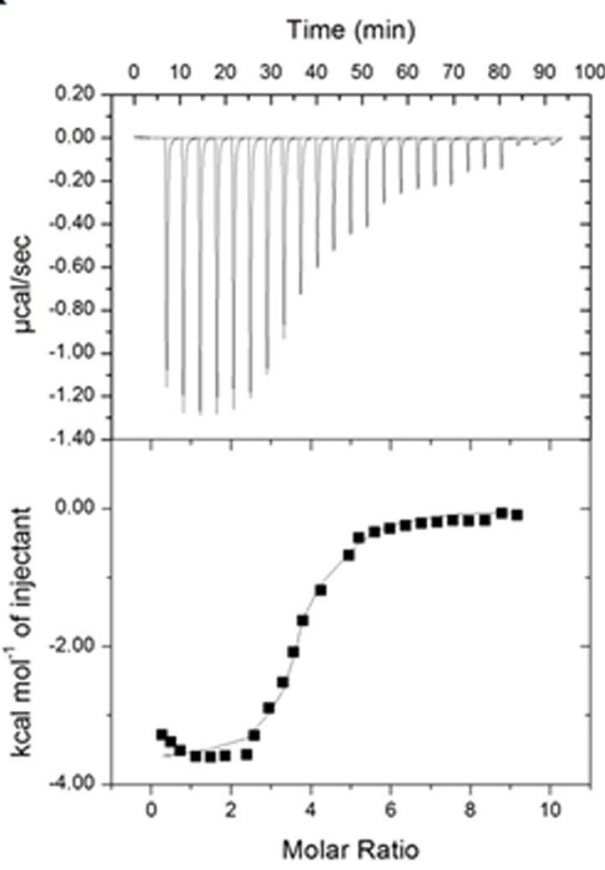

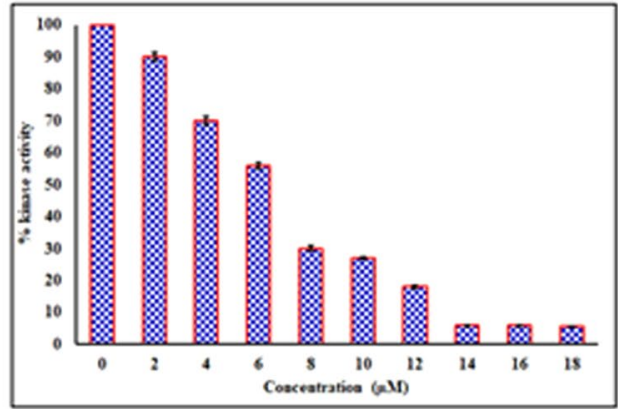

C

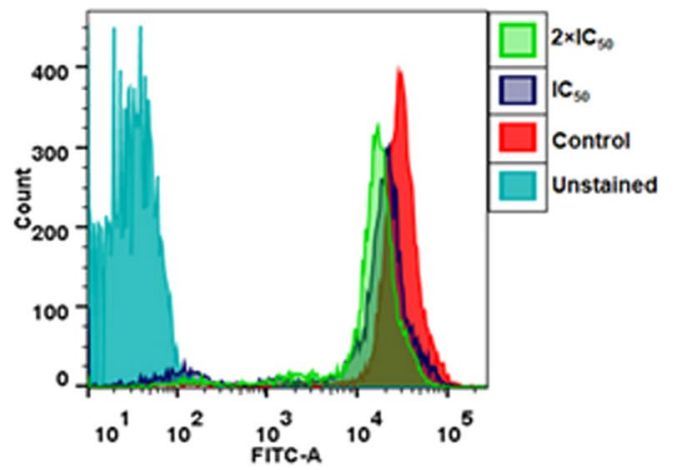

Figure 7. Binding and enzyme inhibition studies of RA with MARK4: (A) ITC profile of RA with MARK4. (Top) Raw data plot of heat produced against time for the titration of $500 \mu \mathrm{M}$ RA into $20 \mu \mathrm{M}$ MARK4. The bottom panel shows resultant binding isotherm prior to the integration of peak area and normalization to yield a plot of molar enthalpy change against RA-MARK4 ratio. (B) ATPase inhibition assay of MARK4 with increasing concentration of RA $(0-18 \mu \mathrm{M})$. The activity of native MARK4 was taken as $100 \%$ for reference. (C) Tau phosphorylation studies of MARK4 with RA. Typical flow cytometry histogram of SH-SY5Y cells obtained from the phosphorylated anti-tau staining, each curve denotes the phosphorylation status of tau in cells with RA treatments as revealed in the inset.

forces in this interaction are van der Waals and hydrogen bonding ${ }^{67}$. All these finding complement our molecular docking observations which suggested that hydrogen bonding and van der Waals interactions were prevalent in MARK4-RA complex.

Measurements of conformational changes. Many studies have shown a little change in the secondary structure of MARK4 by ligand binding. It is evident from Figure SII that after binding of RA to MARK4, there is a slight change in the intensity of the dichroic signal of far-UV CD. This loss in the far-UV CD signal of MARK4-RA as compared to native MARK4 was not much significant. However, a slight loss in the $\alpha$-helical structure of MARK4 upon RA binding was observed.

Isothermal titration calorimetry. Fluorescence binding studies were further complemented by ITC measurements. Figure 7A shows an isotherm of MARK4 titrated with RA. The upper panel corresponds to raw data obtained due to consecutive injection of RA to MARK4. Binding curves obtained after subtracting dilution heat of both ligands and protein are depicted in the bottom panel. The final figure was obtained using Micro Cal VP-ITC Origin 8.0. The obtained isotherm of ITC advocates the binding of RA with MARK4.

Various studies reported the difference in values of thermodynamic parameters as obtained from fluorescence spectroscopy and ITC and this is because ITC measures a global change in the thermodynamic property whereas fluorescence spectroscopy taking into consideration only the local changes around the fluorophore $(\text { Trp-214 })^{68}$. Different thermodynamic parameters obtained for MARK4-RA interaction are as follows: $K_{\mathrm{a}}=$ $4.40 \times 10^{6} \pm 1.34 \times 10^{6} \mathrm{M}^{-1}, \Delta H=-1.69 \times 10^{4} \pm 392.5 \mathrm{kcal} \mathrm{mol}^{-1}$ and $\Delta S=-26.4 \mathrm{cal} . \mathrm{mol}^{-1} \mathrm{~K}^{-1}$. Interestingly, our ITC results correlate with fluorescence binding results, which also suggested that the reaction of MARK4-RA is an exothermic and enthalpy driven process.

Enzyme inhibition and tau-phosphorylation assays. To evaluate the inhibitory potential of RA on the activity of MARK4, enzyme assay was carried out with varying concentrations of RA (0-18 $\mu$ M) (Fig. 7B). The kinase activity of MARK4 is quantified and plotted as percent inhibition compared to the activity of native MARK4, taken as $100 \%$ for reference in the absence of RA. There was a decrease in MARK4 activity with increasing concentration of RA, in a dose-dependent manner (Fig. 7B). $\mathrm{IC}_{50}$ value represents the concentration at which a substance exerts half of its maximal inhibitory effect. ATPase inhibition result showed that RA inhibits 50\% activity of MARK4 at $6.20 \mu \mathrm{M}$, thus $\mathrm{IC}_{50}$ of RA was found to be $6.204 \mu \mathrm{M}$ employing AAT Bioquest calculator. These results suggested that RA is a potent inhibitor of MARK4 validating the molecular docking analysis which 
A

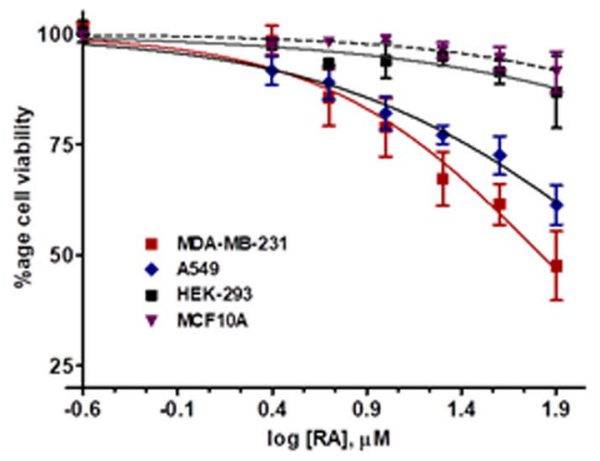

B

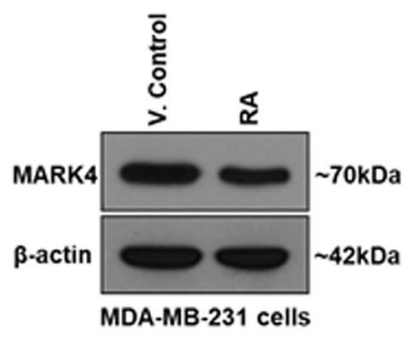

C

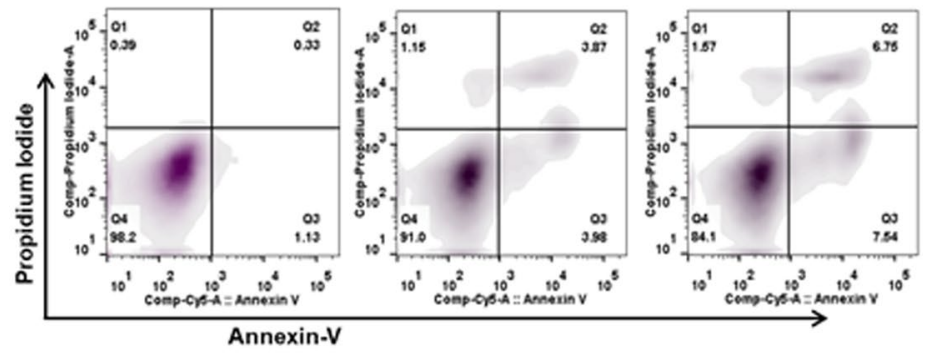

D

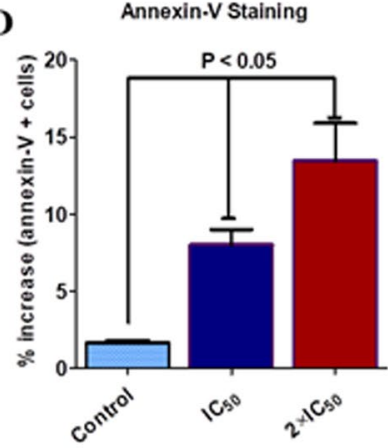

Figure 8. Cell viability, protein expression and apoptosis studies of RA. (A) Cell viability studies of RA with MDA-MB-231, A549, and HEK-293 cells. Each data point shows the mean \pm SD from $n=3$. (B) Protein expression studies of MARK4 in MDA-MB-231 RA cells treated/vehicle-treated cells. Cells were treated with $\mathrm{IC}_{50}$ concentration of RA for $48 \mathrm{~h}$ and total protein was isolated. The expression of the desired protein was accessed using immunoblotting. Raw images of immunoblotting are provided in the supplementary information. (C) Apoptosis induction studies of RA on MDA-MB-231 cells treated with $\mathrm{IC}_{50}$ and $2 \times \mathrm{IC}_{50}$ concentrations for $72 \mathrm{~h}$ using Annexin-V/PI staining. (C) Graphical representation of the percent apoptotic cells obtained from Annexin-V/PI staining for triplicate measurements \pm SD.

also suggested that RA interacts with functionally important residues of MARK4. Thus, it can be concluded that RA strongly binds to the active site pocket residues thereby inhibiting MARK4.

RA potentially inhibited MARK4 activity as suggested by kinase inhibition assay. We further extended our study to see the MARK4 inhibition potential of RA in a cell-based system using tau phosphorylation assay ${ }^{16,20}$. RA treated cells were analyzed for tau-phosphorylation using flow cytometry. We found that RA treatment induces a shift in histogram towards lower value (left of untreated control), suggesting that RA treatment decreases phosphorylation of tau as compared to untreated cells (Fig. 7C). Interestingly, decrease in phosphorylation was noticed in a dose-dependent manner. Overall, tau-phosphorylation results showed that RA inhibited MARK4, as tau is the main substrate of it $^{12,16}$.

Cell proliferation and protein expression studies. RA inhibited MARK4, as overexpression of MARK4 found to be associated with the proliferation and growth of different cancer cells, so the cancer cell growth inhibition studies were carried out. Cell proliferation studies have been performed on MDA-MB-231 and A549 cells as these cells have high expression of MARK4 and serve as the model cells for MARK4 related anticancer studies $^{16,17}$. We found that RA showed differential cell growth inhibition profile against MDA-MB-231 and A549 cells (Fig. 8A). Cell viability studies suggested that treatment of RA significantly inhibited the growth of MDA-MB-231 and A549. To further see the cytotoxic effect of RA on the growth of non-cancer cells, similar studies were carried out on HEK293 and MCF10A cells. The result showed that RA did not affect the viability of HEK293 and MCF-10A cells (Fig. 8A). These results suggested that in the studied concentration range RA has no cytotoxic activity for non-cancer cells, on the other hand, they have significant cytotoxicity for cancer cells. These results are consistent with the previous reports of cytotoxicity for RA performed on similar or other cancer cells $s^{69,70}$. Subsequently, we have studied the effect of RA on the protein expression level of MARK4. For these experiments, MDA-MB-231 cells were treated with $\mathrm{IC}_{50}$ concentration of RA for $48 \mathrm{~h}$ and immunoblotting studies were performed. It was found that the treatment of RA decreases the expression of MARK4 (Fig. 8B). These results suggested that RA inhibited the MARK4 at the protein level as well.

Apoptosis studies. MARK4 plays an important role in the growth, progression and apoptotic evasion of cancer cells ${ }^{71}$. Therefore, we question whether the RA induced inhibition of MARK4 has any effect on the apoptosis of cancer cells. To see the apoptotic potential of RA on MDA-MB-231 cells, these cells were treated with $\mathrm{IC}_{50}$ 
and $2 \times \mathrm{IC}_{50}$ concentration of RA for $72 \mathrm{~h}$ and analyzed for apoptosis induction (using annexin-V/PI staining). Annexin-V staining results showed that RA induces apoptosis in MDA-MB-231 cells in a dose-dependent manner (Fig. 8B). RA induces apoptosis in $9.0 \%$ and $15.90 \%$ of MDA-MB-231 cells at $\mathrm{IC}_{50}$ and $2 \times \mathrm{IC}_{50}$ concentration, respectively compared to control (Fig. $8 \mathrm{C}$ ). Taken together, the results of cell viability and apoptosis studies suggested that RA inhibited the growth of selected cancer cells and induces apoptosis.

\section{Conclusion}

Our present work provided atomistic insights into the binding mechanism of RA to MARK4. In addition, inhibition of MARK4 by RA provides a newer avenue for cancer treatment as its expression is found to be enhanced in many cancers This study proved that RA may be employed as potent MARK4 inhibitor to control cancer cell growth and induction of apotosis. The results of our study unveil the potential of RA and RA derivatives-based inhibitors to be implicated in drug discovery process. Finally, our findings provided a platform to use RA or its derivatives as MAKR4 inhibitors for therapeutic management of MARK4 associated diseases.

Received: 24 January 2020; Accepted: 6 May 2020;

Published online: 25 June 2020

\section{References}

1. Shchemelinin, I., Sefc, L. \& Necas, E. Protein kinases, their function and implication in cancer and other diseases. Folia biologica 52, 81 (2006).

2. Noble, M. E., Endicott, J. A. \& Johnson, L. N. Protein kinase inhibitors: insights into drug design from structure. Science 303, 1800-1805 (2004).

3. Cohen, P. Protein kinases-the major drug targets of the twenty-first century? Nat. Rev. Drug. discovery 1, 309 (2002).

4. Arash, E. H., Shiban, A., Song, S. \& Attisano, L. MARK4 inhibits Hippo signaling to promote proliferation and migration of breast cancer cells. EMBO Rep. 18, 420-436 (2017).

5. Sun, W. et al. Attenuation of synaptic toxicity and MARK4/PAR1-mediated Tau phosphorylation by methylene blue for Alzheimer's disease treatment. Sci. Rep. 6, 34784 (2016).

6. Farese, R. V., Sajan, M. P. \& Standaert, M. L. Insulin-sensitive protein kinases (atypical protein kinase C and protein kinase B/Akt): actions and defects in obesity and type II diabetes. Exp. Biol. Med. 230, 593-605 (2005).

7. Nandipati, K. C., Subramanian, S. \& Agrawal, D. K. Protein kinases: mechanisms and downstream targets in inflammation-mediated obesity and insulin resistance. Mol. Cell. Biochem. 426, 27-45 (2017).

8. Cho, J. H. \& Johnson, G. V. Primed phosphorylation of tau at Thr231 by glycogen synthase kinase $3 \beta$ (GSK3 $\beta$ ) plays a critical role in regulating tau's ability to bind and stabilize microtubules. J. neurochemistry 88, 349-358 (2004).

9. Beghini, A. et al. The neural progenitor-restricted isoform of the MARK4 gene in 19q13. 2 is upregulated in human gliomas and overexpressed in a subset of glioblastoma cell lines. Oncogene 22, 2581 (2003).

10. Tian, L., Wen, A., Dong, S. \& Yan, P. Molecular Characterization of Microtubule Affinity-Regulating Kinase4 from Sus scrofa and Promotion of Lipogenesis in Primary Porcine Placental Trophoblasts. Int. J. Mol. Sci. 20, 1206 (2019).

11. Liu, Z. et al. Mark4 promotes oxidative stress and inflammation via binding to PPAR $\gamma$ and activating NF- $\mathrm{B}$ pathway in mice adipocytes. Sci. Rep. 6, 21382 (2016).

12. Trinczek, B., Brajenovic, M., Ebneth, A. \& Drewes, G. MARK4 is a novel microtubule-associated proteins/microtubule affinityregulating kinase that binds to the cellular microtubule network and to centrosomes. J. Biol. Chem. 279, 5915-5923, https://doi. org/10.1074/jbc.M304528200 (2004).

13. Tang, E. I. et al. Microtubule affinity-regulating kinase 4 (MARK4) is a component of the ectoplasmic specialization in the rat testis. Spermatogenesis 2, 117-126 (2012).

14. Li, L. \& Guan, K.-L. Microtubule-associated protein/microtubule affinity-regulating kinase 4 (MARK4) is a negative regulator of the mammalian target of rapamycin complex 1 (mTORC1). J. Biol. Chem. 288, 703-708 (2013).

15. Marx, A., Nugoor, C., Panneerselvam, S. \& Mandelkow, E. Structure and function of polarity-inducing kinase family MARK/Par-1 within the branch of AMPK/Snf1-related kinases. FASEB J. 24, 1637-1648 (2010).

16. Khan, P. et al. Identification of alpha-Mangostin as a Potential Inhibitor of Microtubule Affinity Regulating Kinase 4. J. Nat. products 82, 2252-2261, https://doi.org/10.1021/acs.jnatprod.9b00372 (2019).

17. Pardo, O. E. et al. miR-515-5p controls cancer cell migration through MARK4 regulation. EMBO Rep. 17, 570-584, https://doi. org/10.15252/embr.201540970 (2016).

18. Sun, C. et al. Inactivation of MARK4, an AMP-activated protein kinase (AMPK)-related kinase, leads to insulin hypersensitivity and resistance to diet-induced obesity. J. Biol. Chem. 287, 38305-38315 (2012).

19. Feng, M., Tian, L., Gan, L., Liu, Z. \& Sun, C. Mark4 promotes adipogenesis and triggers apoptosis in 3T3-L1 adipocytes by activating JNK1 and inhibiting p38MAPK pathways. Biol. Cell 106, 294-307 (2014).

20. Khan, P. et al. Elucidation of dietary polyphenolics as potential inhibitor of microtubule affinity regulating kinase 4: in silico and in vitro studies. Sci. Rep. 7, 9470, https://doi.org/10.1038/s41598-017-09941-4 (2017).

21. Naz, F. et al. Investigation of molecular mechanism of recognition between citral and MARK4: A newer therapeutic approach to attenuate cancer cell progression. Int. J. Biol. macromolecules 107, 2580-2589 (2018).

22. Jenardhanan, P., Mannu, J. \& Mathur, P. P. The structural analysis of MARK4 and the exploration of specific inhibitors for the MARK family: a computational approach to obstruct the role of MARK4 in prostate cancer progression. Mol. Biosyst. 10, 1845-1868 (2014).

23. Gupta, P. et al. Evaluation of ellagic acid as an inhibitor of sphingosine kinase 1: A targeted approach towards anticancer therapy. Biomed Pharmacother 118, 109245, S0753-3322(19)32965-8 (2019).

24. Dahiya, R. et al. Investigation of inhibitory potential of quercetin to the pyruvate dehydrogenase kinase 3: Towards implications in anticancer therapy. Int J Biol Macromol 136, 1076-1085, S0141-8130(19)33270-2 (2019).

25. Gulzar, M. et al. Binding mechanism of caffeic acid and simvastatin to the integrin linked kinase for therapeutic implications: a comparative docking and MD simulation studies. J Biomol Struct Dyn 37, 4327-4337, https://doi.org/10.1080/07391102.2018.1546621 (2019).

26. Sikander, M. et al. Novel Mechanistic Insight into the Anticancer Activity of Cucurbitacin D against Pancreatic Cancer (Cuc D Attenuates Pancreatic Cancer). Cells 9, 103 (2019).

27. Kaul, R., Risinger, A. L. \& Mooberry, S. L. Microtubule-Targeting Drugs: More than Antimitotics. J. Nat. products 82, 680-685 (2019).

28. Hernández-Bolio, G. I., Ruiz-Vargas, J. A. \& Peña-Rodrîguez, L. M. Natural Products from the Yucatecan Flora: Structural Diversity and Biological Activity. J. Nat. products 82, 647-656 (2019).

29. Takamatsu, S. et al. Marine natural products as novel antioxidant prototypes. J. Nat. products 66, 605-608 (2003).

30. Guerra, A. R., Duarte, M. F. \& Duarte, I. F. Targeting tumor metabolism with plant-derived natural products: Emerging trends in cancer therapy. J. Agric. food Chem. 66, 10663-10685 (2018). 
31. Lv, H.-N. et al. Anti-inflammatory coumarin and benzocoumarin derivatives from Murraya alata. J. Nat. products 78, 279-285 (2015).

32. Liu, B.-Y. et al. Anti-inflammatory prenylated phenylpropenols and coumarin derivatives from Murraya exotica. J. Nat. products 81 , 22-33 (2018)

33. Domitrović, R. et al. Rosmarinic acid ameliorates acute liver damage and fibrogenesis in carbon tetrachloride-intoxicated mice. Food Chem. Toxicol. 51, 370-378 (2013).

34. Chu, X. et al. Effects of a natural prolyl oligopeptidase inhibitor, rosmarinic acid, on lipopolysaccharide-induced acute lung injury in mice. Molecules 17, 3586-3598 (2012).

35. Osakabe, N. et al. Anti-inflammatory and anti-allergic effect of rosmarinic acid (RA); inhibition of seasonal allergic rhinoconjunctivitis (SAR) and its mechanism. Biofactors 21, 127-131 (2004).

36. Moon, D.-O., Kim, M.-O., Lee, J.-D., Choi, Y. H. \& Kim, G.-Y. Rosmarinic acid sensitizes cell death through suppression of TNF$\alpha$-induced NF- $\mathrm{B}$ B activation and ROS generation in human leukemia U937 cells. Cancer Lett. 288, 183-191 (2010).

37. Moreno, S., Scheyer, T., Romano, C. S. \& Vojnov, A. A. Antioxidant and antimicrobial activities of rosemary extracts linked to their polyphenol composition. Free. Radic. Res. 40, 223-231 (2006).

38. Ono, K. et al. Phenolic compounds prevent amyloid $\beta$-protein oligomerization and synaptic dysfunction by site-specific binding. $J$. Biol. Chem. 287, 14631-14643 (2012).

39. Xavier, C. P., Lima, C. F., Fernandes-Ferreira, M. \& Pereira-Wilson, C. Salvia fruticosa, Salvia officinalis, and rosmarinic acid induce apoptosis and inhibit proliferation of human colorectal cell lines: the role in MAPK/ERK pathway. Nutr. cancer 61, 564-571 (2009).

40. Xu, Y., Xu, G., Liu, L., Xu, D. \& Liu, J. Anti-invasion effect of rosmarinic acid via the extracellular signal-regulated kinase and oxidation-reduction pathway in Ls174-T cells. J. Cell. Biochem. 111, 370-379 (2010).

41. Greenhough, A. et al. The COX-2/PGE 2 pathway: key roles in the hallmarks of cancer and adaptation to the tumour microenvironment. Carcinogenesis 30, 377-386 (2009).

42. Scheckel, K. A., Degner, S. C. \& Romagnolo, D. F. Rosmarinic acid antagonizes activator protein-1-dependent activation of cyclooxygenase-2 expression in human cancer and nonmalignant cell lines. J. Nutr. 138, 2098-2105 (2008).

43. Trott, O. \& Olson, A. J. AutoDock Vina: improving the speed and accuracy of docking with a new scoring function, efficient optimization, and multithreading. J. computational Chem. 31, 455-461 (2010).

44. Dassault Systèmes BIOVIA, Discovery Studio Modeling Environment, San Diego: Dassault Systèmes, (2017).

45. Schrodinger, L. The PyMOL molecular graphics system. Version 1, 0 (2010).

46. Mohammad, T. et al. Identification and evaluation of bioactive natural products as potential inhibitors of human microtubule affinity-regulating kinase 4 (MARK4). J. Biomolecular Structure Dyn. 37, 1813-1829 (2019).

47. Sack, J. S. et al. Crystal structure of microtubule affinity-regulating kinase 4 catalytic domain in complex with a pyrazolopyrimidine inhibitor. Acta Crystallogr. Sect. F: Struct. Biol. Commun. 72, 129-134 (2016).

48. Pronk, S. et al. GROMACS 4.5: a high-throughput and highly parallel open source molecular simulation toolkit. Bioinformatics 29, 845-854, https://doi.org/10.1093/bioinformatics/btt055btt055 (2013).

49. Oostenbrink, C., Villa, A., Mark, A. E. \& van Gunsteren, W. F. A biomolecular force field based on the free enthalpy of hydration and solvation: the GROMOS force-field parameter sets 53A5 and 53A6. J. Comput. Chem. 25, 1656-1676, https://doi.org/10.1002/ jcc.20090 (2004).

50. Schuttelkopf, A. W. \& van Aalten, D. M. PRODRG: a tool for high-throughput crystallography of protein-ligand complexes. Acta Crystallogr. D. Biol. Crystallogr 60, 1355-1363, https://doi.org/10.1107/S0907444904011679 (2004).

51. Frisc, M. J. et al., Gaussian 16, Revision. Gaussian, Inc., Wallingford CT, 2016.

52. Zielkiewicz, J. Structural properties of water: comparison of the SPC, SPCE, TIP4P, and TIP5P models of water. J. Chem. Phys. 123, 104501, https://doi.org/10.1063/1.2018637 (2005).

53. Kumari, R., Kumar, R. \& Lynn, A. g_mmpbsa-a GROMACS tool for high-throughput MM-PBSA calculations. J. Chem. Inf. Model. 54, 1951-1962, https://doi.org/10.1021/ci500020m (2014).

54. Naz, F., Sami, N., Islam, A., Ahmad, F. \& Hassan, M. I. Ubiquitin-associated domain of MARK4 provides stability at physiological pH. Int. J. Biol. macromolecules 93, 1147-1154 (2016).

55. Naz, F. et al. Cloning, expression, purification and refolding of microtubule affinity-regulating kinase 4 expressed in Escherichia coli. Appl. Biochem. Biotechnol. 172, 2838-2848 (2014).

56. Khan, P. et al. Luminol-based chemiluminescent signals: clinical and non-clinical application and future uses. Appl. Biochem. Biotechnol. 173, 333-355 (2014).

57. Chi, Z. \& Liu, R. Phenotypic characterization of the binding of tetracycline to human serum albumin. Biomacromolecules 12, 203-209 (2010).

58. Queen, A., Khan, P., Idrees, D., Azam, A. \& Hassan, M. I. Biological evaluation of p-toluene sulphonylhydrazone as carbonic anhydrase IX inhibitors: An approach to fight hypoxia-induced tumors. Int. J. Biol. Macromol. 106, 840-850 (2018). S01418130(17)32590-4.

59. Khan, N. S. et al. Thienopyrimidine-Chalcone Hybrid Molecules Inhibit Fas-Activated Serine/Threonine Kinase: An Approach To Ameliorate Antiproliferation in Human Breast Cancer Cells. Mol. Pharm. 15, 4173-4189, https://doi.org/10.1021/acs. molpharmaceut.8b00566 (2018).

60. Peerzada, M. N., Khan, P., Ahmad, K., Hassan, M. I. \& Azam, A. Synthesis, characterization and biological evaluation of tertiary sulfonamide derivatives of pyridyl-indole based heteroaryl chalcone as potential carbonic anhydrase IX inhibitors and anticancer agents. Eur J Med Chem 155, 13-23, https://doi.org/1016/j.ejmech.2018.05.034 (2018).

61. Soares, S., Mateus, N. \& De Freitas, V. Interaction of different polyphenols with bovine serum albumin (BSA) and human salivary $\alpha$-amylase (HSA) by fluorescence quenching. J. Agric. Food Chem. 55, 6726-6735 (2007).

62. Klajnert, B., Stanisławska, L., Bryszewska, M. \& Pałecz, B. Interactions between PAMAM dendrimers and bovine serum albumin. Biochimica et. Biophysica Acta-Proteins Proteom. 1648, 115-126 (2003).

63. Saquib, Q. et al. Fungicide methyl thiophanate binding at sub-domain IIA of human serum albumin triggers conformational change and protein damage. Int. J. Biol. macromolecules 47, 60-67 (2010).

64. Shamsi, A., Ahmed, A. \& Bano, B. Probing the interaction of anticancer drug temsirolimus with human serum albumin: Molecular docking and spectroscopic insight. J. Biomolecular Structure Dyn. 36, 1479-1489 (2018).

65. Khan, S. N. et al. Interaction of mitoxantrone with human serum albumin: Spectroscopic and molecular modeling studies. Eur. J. Pharm. Sci. 35, 371-382 (2008).

66. Cahyana, Y. \& Gordon, M. H. Interaction of anthocyanins with human serum albumin: Influence of $\mathrm{pH}$ and chemical structure on binding. Food Chem. 141, 2278-2285 (2013).

67. Ross, P. D. \& Subramanian, S. Thermodynamics of protein association reactions: forces contributing to stability. Biochemistry 20 , 3096-3102 (1981).

68. Rehman, M. T., Shamsi, H. \& Khan, A. U. Insight into the binding mechanism of imipenem to human serum albumin by spectroscopic and computational approaches. Mol. Pharmaceutics 11, 1785-1797 (2014).

69. Yesil-Celiktas, O., Sevimli, C., Bedir, E. \& Vardar-Sukan, F. Inhibitory effects of rosemary extracts, carnosic acid and rosmarinic acid on the growth of various human cancer cell lines. Plant Foods Hum Nutr 65, 158-163, https://doi.org/10.1007/s11130-010-0166-4. 
70. Li, H. et al. Effect of rosmarinic acid from Sarcandra glabra in inhibiting proliferation and migration and inducing apoptosis of MDA-MB-231 cells via regulation of expressions of Bcl-2 and Bax. Zhongguo Zhong Yao Za Zhi 43, 3335-3340, https://doi. org/10.19540/j.cnki.cjcmm.20180508.001.

71. Naz, F., Anjum, F., Islam, A., Ahmad, F. \& Hassan, M. I. Microtubule affinity-regulating kinase 4: structure, function, and regulation. Cell Biochem. Biophys. 67, 485-499, https://doi.org/10.1007/s12013-013-9550-7 (2013).

\section{Acknowledgements}

S.A. and A.Q. thank to the Indian Council of Medical Research (ICMR) for the award of Senior Research Fellowship (45/37/2019-BIO/BMS and 45/63/2018-PHA/BMS/OL). MFA and AH thank to the King Saud University for financial support (Grant number RSP-2019-122). Authors sincerely thank to the Department of Science and Technology, Government of India for the FIST support (FIST program No. SR/FST/LSI-541/2012). This work is supported by the Science and Engineering Research Board, Department of Science and Technology, Government of India (Grant No. EMR/2015/002372).

\section{Author contributions}

All authors have read and agreed to publish the current version of the manuscript. Conceptualization, S.A., A.S. and M.I.H.; methodology, S.A., M.S. and A.S.; software, M.S., P.K. and A.H.; validation, G.M.H, A.Q. and M.I.H.; formal analysis, M.F.A., M.S., P.K., A.H.; investigation, S.A., A.Q., M.S. and A.S.; resources, M.F.A. and A.H.; data curation, M.S., G.M.H. and A.I.; writing-original draft preparation, S.A., A.S. and M.I.H.; writing-review and editing, F.A., M.F.A. and M.I.H.; visualization, A.H., P.K., M.S., and F.A.; supervision, M.F.A. and M.I.H; project administration, F.A. and M.I.H; funding acquisition, M.F.A. and M.I.H.

\section{Competing interests}

The authors declare no competing interests.

\section{Additional information}

Supplementary information is available for this paper at https://doi.org/10.1038/s41598-020-65648-z.

Correspondence and requests for materials should be addressed to M.I.H.

Reprints and permissions information is available at www.nature.com/reprints.

Publisher's note Springer Nature remains neutral with regard to jurisdictional claims in published maps and institutional affiliations.

(c) (i) Open Access This article is licensed under a Creative Commons Attribution 4.0 International License, which permits use, sharing, adaptation, distribution and reproduction in any medium or format, as long as you give appropriate credit to the original author(s) and the source, provide a link to the Creative Commons license, and indicate if changes were made. The images or other third party material in this article are included in the article's Creative Commons license, unless indicated otherwise in a credit line to the material. If material is not included in the article's Creative Commons license and your intended use is not permitted by statutory regulation or exceeds the permitted use, you will need to obtain permission directly from the copyright holder. To view a copy of this license, visit http://creativecommons.org/licenses/by/4.0/.

(c) The Author(s) 2020 